Corrigendum

\title{
Corrigendum to "A Simple Method to Assess In Vivo Proliferation in Lung Vasculature with EdU: The Case of MMC-Induced PVOD in Rat"
}

\author{
Fabrice Antigny, ${ }^{1,2,3}$ Benoît Ranchoux, ${ }^{1,2,3}$ Valérie Nadeau, ${ }^{4}$ Edmund Lau, ${ }^{1,2,3}$ \\ Sébastien Bonnet, ${ }^{4}$ and Frédéric Perros ${ }^{1,2,3,4}$ \\ ${ }^{1}$ Faculté de Médecine, Université Paris-Sud, Le Kremlin-Bicêtre, France \\ ${ }^{2}$ AP-HP, Centre de Référence de l'Hypertension Pulmonaire Sévère, Département Hospitalo-Universitaire (DHU) Thorax Innovation, \\ Service de Pneumologie et Réanimation Respiratoire, Hôpital de Bicêtre, Le Kremlin-Bicêtre, France \\ ${ }^{3}$ UMRS 999, INSERM and Université Paris-Sud, Laboratoire d'Excellence (LabEx) en Recherche sur le Médicament et \\ l'Innovation Thérapeutique (LERMIT), Centre Chirurgical Marie Lannelongue, Le Plessis-Robinson, France \\ ${ }^{4}$ Groupe de Recherche en Hypertension Pulmonaire, Centre de Recherche de l'Institut Universitaire de Cardiologie et \\ de Pneumologie de Québec, QC, Canada
}

Correspondence should be addressed to Fabrice Antigny; antignyfabrice@gmail.com

Received 29 December 2016; Accepted 24 January 2017; Published 6 February 2017

Copyright (C) 2017 Fabrice Antigny et al. This is an open access article distributed under the Creative Commons Attribution License, which permits unrestricted use, distribution, and reproduction in any medium, provided the original work is properly cited.

In the article titled "A Simple Method to Assess In Vivo Proliferation in Lung Vasculature with EdU: The Case of MMC-Induced PVOD in Rat" [1], the authors' first and last names were reversed. The corrected authors' list is shown above.

\section{References}

[1] A. Fabrice, R. Benoît, N. Valérie, E. Lau, B. Sébastien, and P. Frédéric, "A simple method to assess in vivo proliferation in lung vasculature with EdU: the case of MMC-induced PVOD in rat," Analytical Cellular Pathology, vol. 2015, Article ID 326385, 6 pages, 2015. 


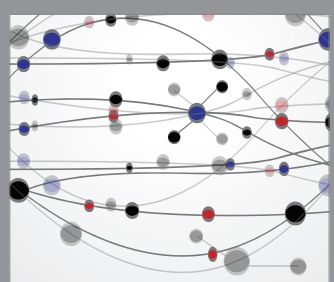

The Scientific World Journal
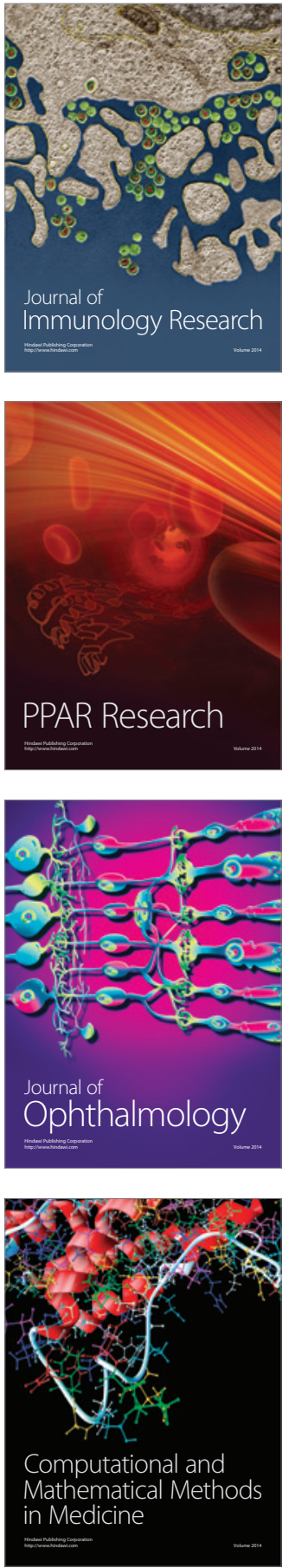

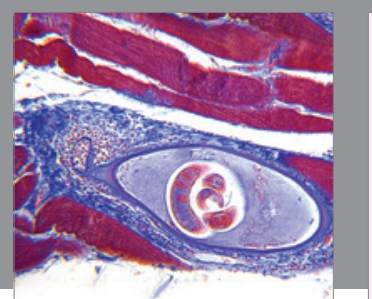

Gastroenterology Research and Practice
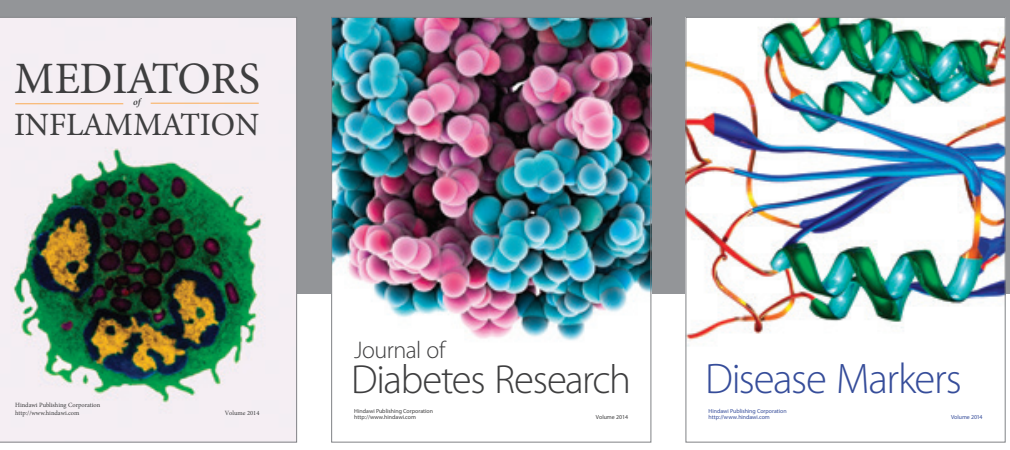

Disease Markers

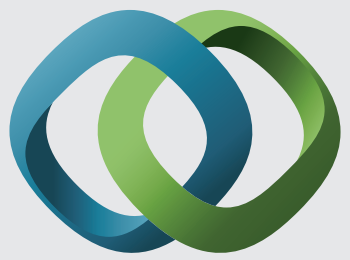

\section{Hindawi}

Submit your manuscripts at

https://www.hindawi.com
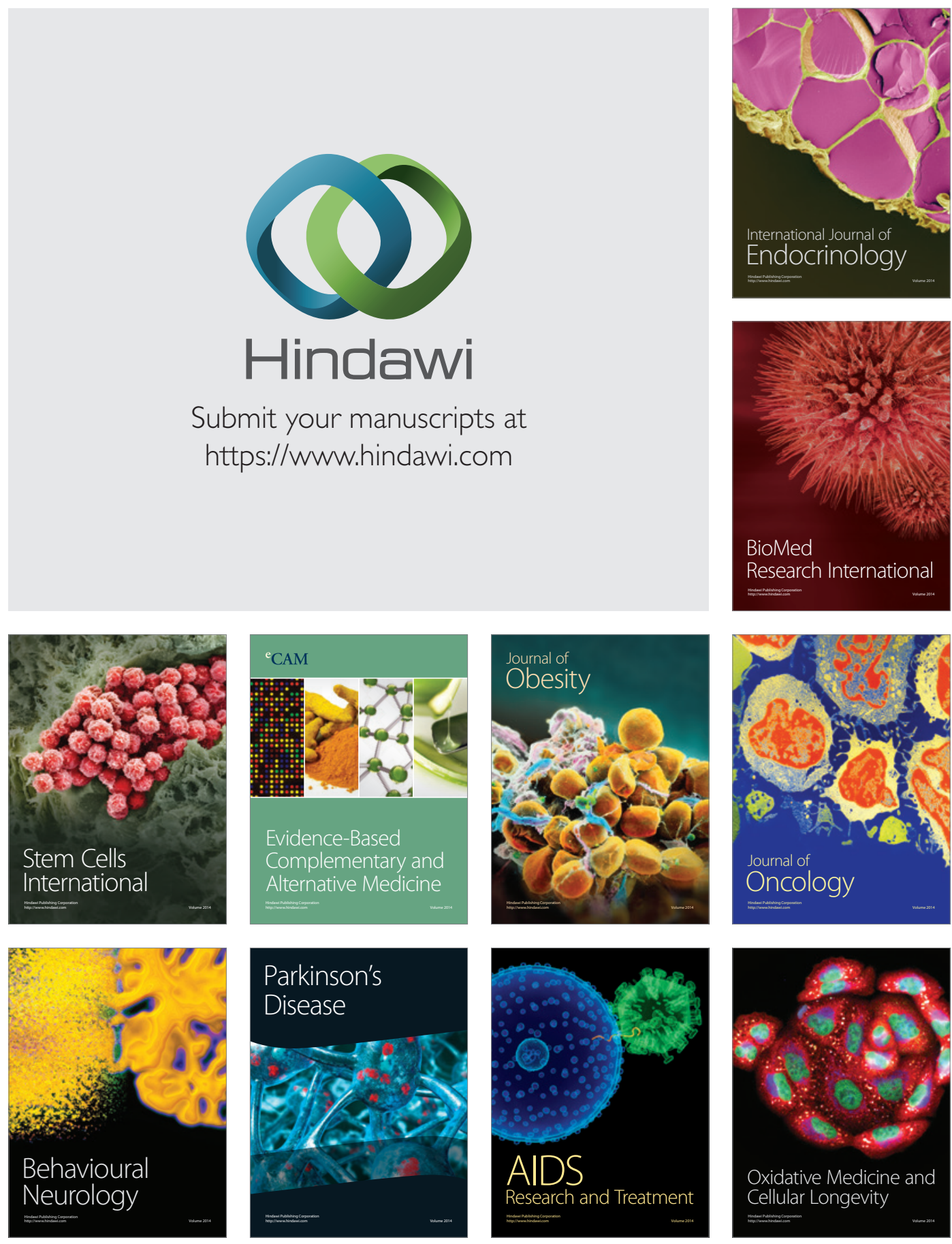